INPLASY

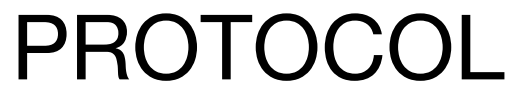

To cite: Cong et al. Effect of sling exercise therapy on balance and walking ability in stroke patients: A Metaanalysis. Inplasy protocol 202140046. doi:

10.37766/inplasy2021.4.0046

Received: 08 April 2021

Published: 08 April 2021

Corresponding author:

Yingshu Cong

995974785@qq.com

Author Affiliation:

Beihua University

Support: Jilin province.

Review Stage at time of this submission: Formal screening of search results against eligibility criteria.

Conflicts of interest:

None declared.

\section{Effect of sling exercise therapy on balance and walking ability in stroke patients: A Meta-analysis}

Cong, $\mathrm{Y}^{1} ; \mathrm{Han}, \mathrm{W}^{2} ;$ Mei, $\mathrm{C}^{3}$.

Review question / Objective: P: stroke patients; I: sling exercise therapy; C: conventional physical therapy; O: Berg balance scale; Fugl-Meyer scale; functional ambulation category scale; S: a randomized controlled trial.

Condition being studied: Stroke patients.

Information sources: Randomized controlled trials about sling exercise therapy improve lower extremity function in stroke patients were recalled from databases of Cochrane Library, MEDLINE, PubMed, EMBASE, CNKI, Wan-fang data, CBM, and VIP.

INPLASY registration number: This protocol was registered with the International Platform of Registered Systematic Review and Meta-Analysis Protocols (INPLASY) on 08 April 2021 and was last updated on 08 April 2021 (registration number INPLASY202140046).

\section{INTRODUCTION}

Review question / Objective: P: stroke patients; I: sling exercise therapy; C: conventional physical therapy; O: Berg balance scale; Fugl-Meyer scale; functional ambulation category scale; $S$ : a randomized controlled trial.
Condition being studied: Stroke patients.

\section{METHODS}

Participant or population: Stroke patients.

Intervention: Sling exercise therapy. 
Comparator: Conventional physical therapy.

Study designs to be included: Randomized controlled trials about sling exercise therapy improve lower extremity function in stroke patients were recalled from databases of Cochrane Library, MEDLINE, PubMed, EMBASE, CNKI, Wan-fang data, CBM. The quality of the trials was evaluated and the data were extracted. Data were analyzed with Revman 5.4 .

Eligibility criteria: 1.Experiment design: randomized controlled trials 2.Subjects: Stroke patients aged $\geq 18$ years 3.Intervention measures: Conventional physical therapy was used in the control group; The experimental group was treated with suspension exercise therapy. 4.Outcomes: Berg balance scale; FuglMeyer scale; functional ambulation category scale.

Information sources: Randomized controlled trials about sling exercise therapy improve lower extremity function in stroke patients were recalled from databases of Cochrane Library, MEDLINE, PubMed, EMBASE, CNKI, Wan-fang data, CBM, and VIP.

Main outcome(s): Berg balance scale; FuglMeyer scale; functional ambulation category scale.

Quality assessment / Risk of bias analysis: The study quality of the included literature was evaluated according to the quality assessment criteria recommended by the Cochrane system evaluator's manual: A: generation of random assignment schemes; B: Whether to hide the distribution scheme; C: Whether to blind the subjects;D: Whether to use the blind method for the evaluator; E: Incomplete result data; F: Selective reporting of results; G: Other risks of bias.

Strategy of data synthesis: The RevMan5.4 software provided by the Cochrane Collaboration was used for Meta-analysis of the extracted data.
Subgroup analysis: 1. Intervention time: less than 15 days; 2 . Intervention time: 15-30 days; 3 . Intervention time: more than 30 days.

Sensitivity analysis: Sensitivity analysis was conducted for trials with too long intervention time or too heavy intervention intensity.

Language: English and Chinese.

Country(ies) involved: China.

Keywords: Stroke; sling exercise therapy; Exercise rehabilitation; balance; walking; meta-analysis.

Contributions of each author:

Author 1 - Yingshu Cong.

Author 2 - Wumo Han.

Author 3 - Chunli Mei. 